\title{
Isolated gastric Kaposi's sarcoma in a non-AIDS/ HIV patient with coexisting collagenous gastritis and microscopic colitis
}

\author{
Andrey P. Kiryukhin ${ }^{1}$, Alexander S. Tertychnyy ${ }^{2}$, Petr L. Shcherbakov ${ }^{3}$, Alexandr S. Inozemtsev ${ }^{4}$, and \\ Olga A. Kolokolnikova ${ }^{5}$
}

\begin{abstract}
${ }^{1}$ Endoscopy Unit, The Second University Clinic, I.M. Sechenov First Moscow State Medical University (Sechenov University), Moscow; ${ }^{2}$ Department of Pathology, I.M. Sechenov First Moscow State Medical University (Sechenov University), Moscow; ${ }^{3}$ Department of Gastroenterology and Endoscopy, Postgraduate Education Academy MEDSI, Moscow; Departments of ${ }^{4}$ Endoscopy and ${ }^{5} \mathrm{Gastroenterology}$ and Biological Therapy, Clinical Hospital-1 MEDSI, Moscow, Russia
\end{abstract}

Received: November 20, 2020 Revised : December 3, 2020 Accepted: December 4, 2020

\section{Correspondence to} Andrey P. Kiryukhin, M.D.

Tel: +7-903-506-6153

Fax: +7-903-506-6153

E-mail: a.p.kiryukhin@gmail.com https://orcid.org/0000-0001-56858784
A 66-year-old non-human immunodeficiency virus (HIV) woman was admitted with recurrent moderate cramp-like abdominal pain, nonbloody diarrhea, periodic nausea, and fatigue. Her medical history included terminal ileitis since 2 years. She had no unhealthy habits and her medication history included mesalazine and methylprednisolone (9 mg daily) for the past 4 months with improved symptoms. Physical examination revealed no skin rash, mild epigastric tenderness on abdominal palpation, and nonpalpable liver and spleen. Laboratory findings disclosed mild anemia and increased levels of fecal calprotectin and C-reactive protein. HIV-1,2 Western blots were negative. Abdominal ultrasonography and con- trast-enhanced computed tomography showed unremarkable findings. Upper gastrointestinal endoscopy revealed atrophy and a reddish, round, and elevated maculopapular lesion with friability in the stomach body (Fig. 1A). Mucosal nodularity with no abnormal capillary vessels was observed throughout the gastric antrum (Fig. 1B). Intramucosal ruptures and hemorrhage without any perforation signs had happened after biopsy (Fig. 1C). She was treated with hemostatic agents and showed positive effects. Helicobacter pylori were not detected. Histopathology demonstrated spindle-cell proliferation in the submucosa (Fig. 2A), emphasized by immunochemistry for $\mathrm{CD}_{31}$ (Fig. 2B) and positivity to human herpesvirus 8 (Fig. $2 \mathrm{C}$ ). Deposition of $>10-\mu \mathrm{m}$ thick
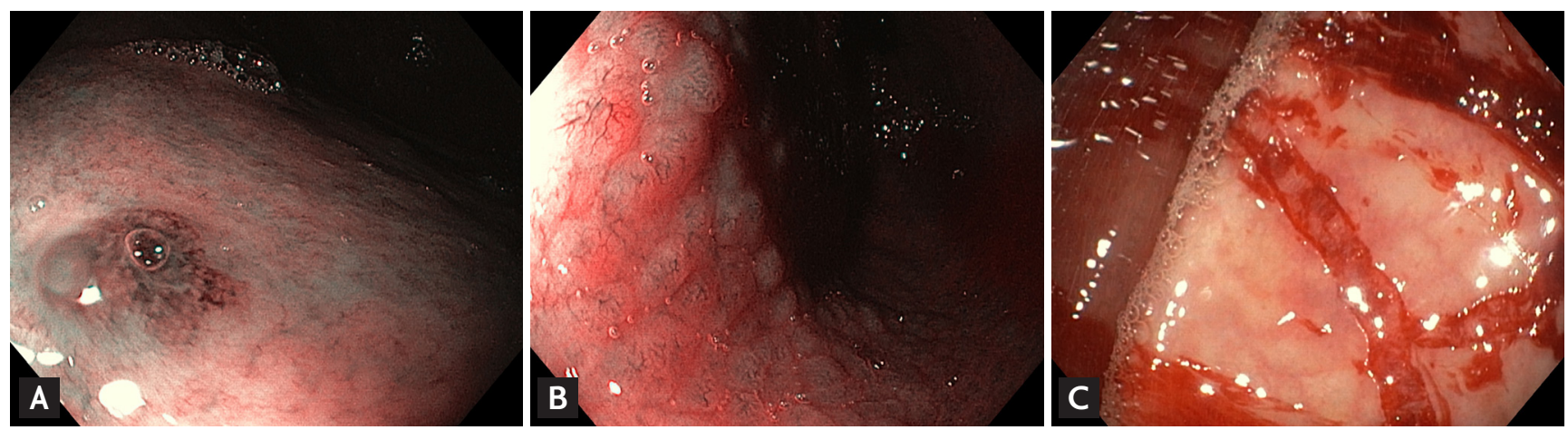

Figure 1. (A) Upper gastrointestinal (GI) endoscopy showing atrophy and a round, elevated maculopapular lesion with friability in the stomach body in narrow-band Imaging mode. (B) Upper GI endoscopy showing mucosal nodularity with no abnormal capillary vessels in the stomach antrum in narrow-band Imaging mode. (C) Upper GI endoscopy showing intramucosal ruptures and hemorrhage after biopsy. 


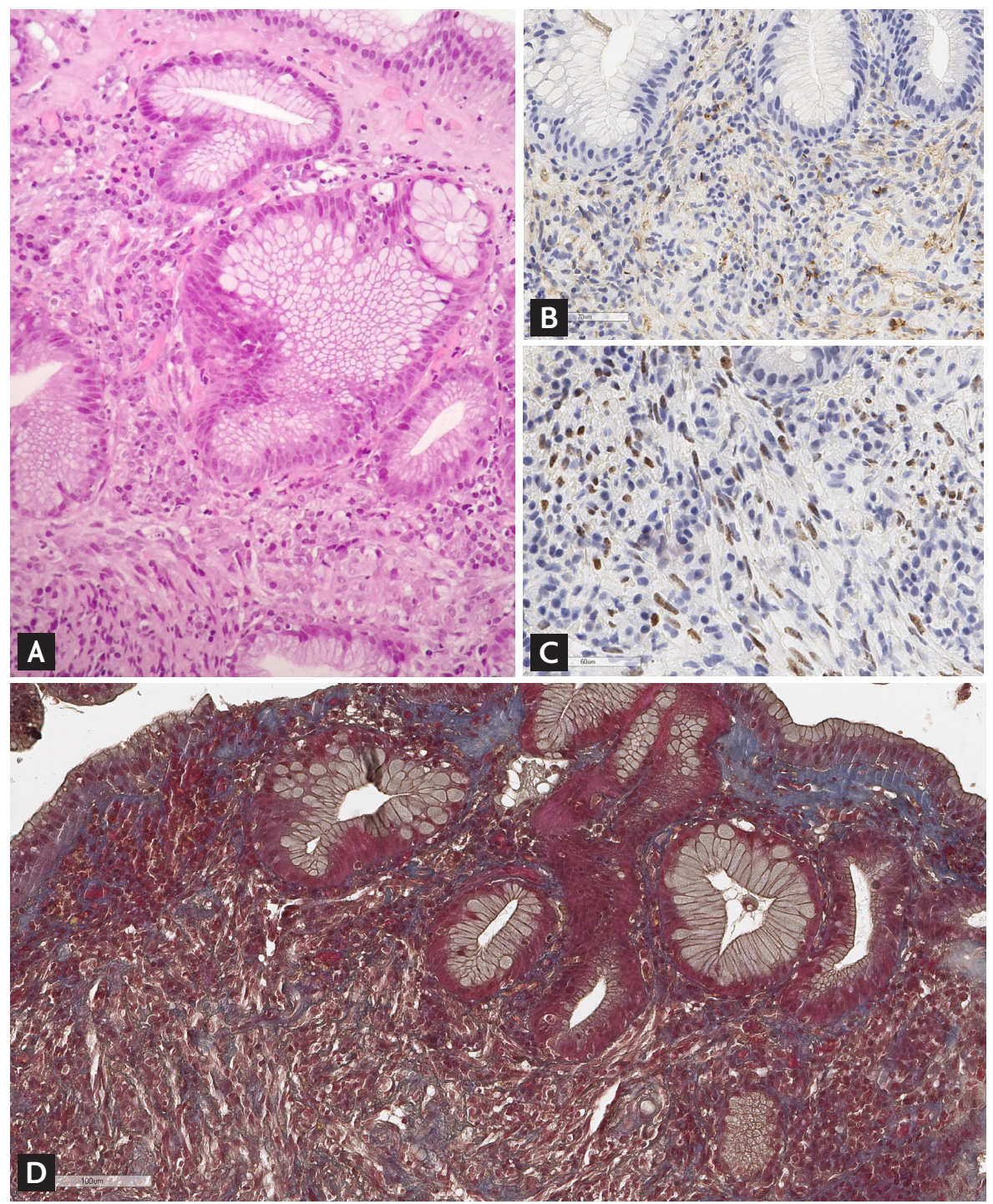

Figure 2.(A) Histopathological section revealed spindle-cell proliferation in the submucosa (H\&E, ×250). (B) Immunohistochemical staining with CD31 showed strong immunopositivity (×250). (C) Immunohistochemical staining with human herpesvirus 8 showed strong positive staining (×250). (D) Histopathological section revealed a deposition of thick collagen bands in the subepithelial layer (positive Masson's trichrome staining, $\times 250$ ). collagen bands was observed in the subepithelial layer, and the specimen was positive for Masson's trichrome staining (Fig. 2D). Colonoscopy and small bowel capsule endoscopy were unremarkable. Histopathology of the colon showed that chronic inflammatory cells had infiltrated in the subepithelial layer with collagen deposition. She was diagnosed with isolated gastric Kaposi's sarcoma, coexisting collagenous gastritis, and microscopic colitis. Treatment with budesonide, esomeprazole, pegylated liposomal doxorubicin was prescribed.

Kaposi's sarcoma is a spindle-cell tumor originating in the vascular endothelium and associated with human herpesvirus 8 infection with an incidence of 1.58/100,000 persons/year in a non-acquired immune deficiency syndrome (AIDS) patient. Gastrointestinal involvement (in
$55 \%$ of cases with an affection of the stomach) may occur without skin lesions and can be asymptomatic, cause gastrointestinal bleeding, or diarrhea. Collagenous gastritis is rare with a poorly understood etiology. Approximately 60 such patients have been reported in English literature. To our knowledge, isolated gastric Kaposi's sarcoma in a non-AIDS patient with coexisting collagenous gastritis and microscopic colitis has not been reported.

Informed consent was obtained from the patient for the publication of this case.

\section{Conflict of interest}

No potential conflict of interest relevant to this article was reported. 